\title{
Structural Disorder and NMR of Quadrupolar Nuclei
}

\author{
Marcin Olszewski ${ }^{1}$
}

Received: 15 July 2018 / Revised: 24 March 2019 / Published online: 17 April 2019

(c) The Author(s) 2019

\begin{abstract}
Growing computational power in connection with an increasing accuracy of nuclear magnetic resonance (NMR) measurements requires precise models for fitting NMR spectra. The occurrence of structural disorder in the material causes an inaccuracy of NMR signals fitted with standard methods. This article presents the calculation of distribution of the quadrupolar coupling constant and asymmetry parameter of the electric field gradient (EFG) tensor in solids with local disorder. In this work, the calculation was made directly from the disturbance of the initial electric charge system surrounding the quadrupolar nucleus without using an approach based on EFG tensor invariants.
\end{abstract}

\section{Introduction}

The precise fitting of powder nuclear magnetic resonance (NMR) spectra of quadrupolar nuclei requires powerful computers. The necessity of integration over all orientation of crystalites gives a spectrum without an analytic representation. In addition to that, the use of the well-known models [1] in connection with a high accuracy of NMR measurements causes a perfect fit not being possible at each attempt [2, 3]. This may be because of many reasons: from some unidentified apparatus errors to an inaccuracy of the physical model. For many compounds, such as glasses with coordination number less or equal to 3 (e.g. boron glasses) [4], nanomaterials [5, 6] and other substances with structural disorder, a similar approach as for powders [1] cannot be used anymore. Random deformations occurring in such materials should be taken into account in high precision measurements.

The basic problem in this situation is to determine a joint distribution of parameters describing quadrupolar interaction. In this work, such a distribution is obtained without using an approach based on electric field gradient (EFG) tensor invariants [7-9]. This approach can be useful for compounds with structural disorder

Marcin Olszewski

marcin.olszewski@usz.edu.pl

1 Faculty of Mathematics and Physics, University of Szczecin, Szczecin, Poland 


\section{EFG Tensor}

The part of the Hamiltonian corresponding to the nuclear quadrupole interaction can be written as $[1,11]$ :

$$
H_{Q}=\frac{e Q}{2 I(2 I-1) \hbar} \boldsymbol{I} \cdot \boldsymbol{V} \cdot \boldsymbol{I}
$$

where $Q$ is the nuclear quadrupole moment and $\boldsymbol{V}$ is the electric field gradient (EFG) tensor with components in the Cartesian coordinates:

$$
V_{i k}=\frac{\partial^{2} \varphi}{\partial x_{i} \partial x_{k}},
$$

where $\varphi$ is the electric field potential at the nucleus. The EFG tensor is symmetric and traceless, thus it has five independent components. In this article the Cartesian coordinates will be used interchangeably $\left(x_{1} \equiv x, x_{2} \equiv y, x_{3} \equiv z\right)$. The components (1) for a discrete charges $q_{n}$ system can be written as:

$$
V_{i k}=\sum_{n} q_{n}\left(3 x_{i}^{(n)} x_{k}^{(n)}-r_{n}^{2} \delta_{i k}\right) r_{n}^{-5}
$$

where $r=\sqrt{x^{2}+y^{2}+z^{2}}$. The EFG tensor can be diagonalized by the suitable choice of a coordinate system. Such a system is called the principal axis system (PAS) in which only two components are independent.

The complete definition of the EFG tensor requires five quantities. Instead of $V_{i k}$ components, the other five are used: $V_{z z}^{\mathrm{PAS}}$, asymmetry parameter $\eta=\left(V_{x x}^{\mathrm{PAS}}-V_{y y}^{\mathrm{PAS}}\right) / V_{z z}^{\mathrm{PAS}}$ and three Euler angles [1, 7] with the convention $\left|V_{z z}^{\mathrm{PAS}}\right| \geqslant\left|V_{y y}^{\mathrm{PAS}}\right| \geqslant\left|V_{x x}^{\mathrm{PAS}}\right|$. As the splitting of nuclear energy levels does not depend on the Euler angles, the occurrence of powder NMR signals is determined by $V_{z z}^{\text {PAS }}$ and $\eta$ only. The superscripts "PAS" can be omitted as long as it will cause no confusion.

\section{Slightly Deformed Charge System}

Let us consider an assembly of discrete electric charges in PAS. The position change of the $N$ th charge of a small vector $\Delta r=(\Delta x, \Delta y, \Delta z)$ makes changes of the EFG tensor components (see Fig. 1).

The new charge system is not in PAS and requires re-diagonalization. This fact makes the problem of impact of $\Delta \boldsymbol{r}$ randomization on $V_{z z}$ and $\eta$ very complicated, because not only diagonal components of the EFG tensor change but also non-zero off-diagonal elements appear.

The rotations $R(\alpha, \beta, \gamma)$ [11] defined for very small Euler angles may by written as (to the first degree terms in Taylor expansion): 
Fig. 1 Position change of the of $N$ th charge of small vector $\Delta \boldsymbol{r}$

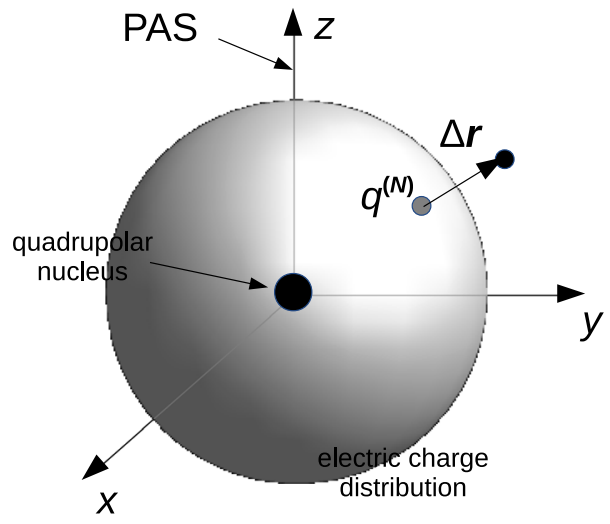

$$
\boldsymbol{R}(\alpha, \beta, \gamma) \approx\left[\begin{array}{ccc}
1 & \alpha+\gamma & -\beta \\
-(\alpha+\gamma) & 1 & 0 \\
\beta & 0 & 1
\end{array}\right]
$$

and it transforms any diagonal matrix in the following way:

$$
\begin{aligned}
\boldsymbol{R}\left[\begin{array}{lll}
a & 0 & 0 \\
0 & b & 0 \\
0 & 0 & c
\end{array}\right] \boldsymbol{R}^{T} & =\left[\begin{array}{ccc}
a-b(\alpha+\gamma)^{2}+c \beta^{2} & (a-b)(\alpha+\gamma) & (c-a) \beta \\
(a-b)(\alpha+\gamma) & b+a(\alpha+\gamma)^{2} & -a \beta(\alpha+\gamma) \\
(c-a) \beta & -a \beta(\alpha+\gamma) & c+a \beta^{2}
\end{array}\right] \\
& \approx\left[\begin{array}{ccc}
a & (a-b)(\alpha+\gamma) & (c-a) \beta \\
(a-b)(\alpha+\gamma) & b & 0 \\
(c-a) \beta & 0 & c
\end{array}\right] .
\end{aligned}
$$

As we can see, to the first degree terms, small rotations do not change the diagonal elements of a matrix; therefore, only the influence of shift of a selected charge on the diagonal components will be taken into account.

In that case, a small shift of $N$ th charge makes a small change of the EFG diagonal components:

$$
\Delta V_{i i} \approx \sum_{k} A_{i k} \Delta x_{k}
$$

where

$$
A_{i k}=\frac{\partial V_{i i}}{\partial x_{k}^{(N)}} .
$$

Further, for convenience, the superscript $(N)$ will be omitted in $x_{k}^{(N)}$ and $r^{(N)}$ for the $N$ th charge. Then, the explicit form of $A_{i k}$ can be written as 


$$
A_{i k}=\frac{3 x_{k}}{r^{5}}\left(1+2 \delta_{i k}-\frac{5 x_{i}^{2}}{r^{2}}\right) .
$$

We can easily check that $\operatorname{det} A=0$, which confirms the traceless property of $\boldsymbol{V}$. No assumption of distribution of variables $\Delta x, \Delta y, \Delta z$ can give the opportunity to obtain a joint distribution of $\Delta V_{x x}, \Delta V_{y y}, \Delta V_{z z}$. Because, if $\Delta V_{x x}, \Delta V_{y y}$ are known, $\Delta V_{z z}$ can be determined. Therefore, obtaining the distribution of $\Delta V_{z z}$ and $\eta$ parameters may be done through finding a joint distribution of $\Delta V_{x x}, \Delta V_{y y}$, which are defined by linear transformation:

$$
\left[\begin{array}{l}
\Delta V_{x x} \\
\Delta V_{y y}
\end{array}\right]=\left[\begin{array}{lll}
A_{11} & A_{12} & A_{13} \\
A_{21} & A_{22} & A_{23}
\end{array}\right]\left[\begin{array}{c}
\Delta x \\
\Delta y \\
\Delta z
\end{array}\right]
$$

\section{Distribution $P_{V}\left(V_{x x}, V_{y y}\right)$}

The knowledge of $P_{x y z}$ distribution of $\Delta x, \Delta y, \Delta z$ shifts is necessary to obtain the distribution $P_{\Delta V}$ for components $\Delta V_{x x}, \Delta V_{y y}$. The assumption that independent but identical Gauss distributions $P_{s}$ with standard deviation $\sigma_{s}$ for each shift is natural due to central limit theorem [12]:

$$
P_{x y z}(\Delta x, \Delta y, \Delta z)=\prod_{i} P_{s}\left(\Delta x_{i}\right)=\frac{1}{(2 \pi)^{3 / 2} \sigma_{s}^{3}} \mathrm{e}^{-\frac{\Delta r^{2}}{2 \sigma_{s}^{2}}} .
$$

Because $P_{x y z}$ is Gaussian and the transformation (2) is linear, $P_{\Delta V}$ is Gaussian too. Thus, we can write directly as follows:

$$
\begin{aligned}
P_{\Delta V}\left(\Delta V_{x x}, \Delta V_{y y}\right)= & \frac{1}{2 \pi \sigma_{x} \sigma_{y} \sqrt{1-\rho^{2}}} \exp \\
& \times\left[-\frac{1}{2\left(1-\rho^{2}\right)}\left(\frac{\Delta V_{x x}^{2}}{\sigma_{x}^{2}}+\frac{\Delta V_{y y}^{2}}{\sigma_{y}^{2}}-2 \rho \frac{\Delta V_{x x} \Delta V_{y y}}{\sigma_{x} \sigma_{y}}\right)\right] .
\end{aligned}
$$

We can use this distribution with any three parameters $\sigma_{x}, \sigma_{y}, \rho$ that are some functions of structural parameters, but their explicit knowledge allows to find dependencies between them and as a result to reduce the number of variables at later numerical modeling. Because the relation (2) maps three to two variables, density transformation can be done using helper coordinate $\Delta V_{h}$. Then the new distribution $P_{\Delta V h}\left(\Delta V_{x x}, \Delta V_{y y}, \Delta V_{h}\right)$ can be obtained through the Jacobian, and distribution $P_{\Delta V}$ can be determined by integration out helper coordinate $\Delta V_{h}$. Let us assume that

$$
\Delta \boldsymbol{V}_{h}=\bar{A} \Delta \boldsymbol{r}^{T},
$$

where 


$$
\overline{\boldsymbol{A}}=\left[\begin{array}{ccc}
A_{11} & A_{12} & A_{13} \\
A_{21} & A_{22} & A_{23} \\
0 & 0 & \frac{6 z}{r^{5}}
\end{array}\right]
$$

and

$$
\Delta \boldsymbol{V}_{h}=\left[\begin{array}{c}
\Delta V_{x x} \\
\Delta V_{y y} \\
\Delta V_{h}
\end{array}\right]
$$

Then,

$$
P_{\Delta V h}\left(\Delta V_{h}^{T}\right)=P_{x y z}\left(\widetilde{A} \Delta r^{T}\right) \cdot J,
$$

where $J=\left|\frac{\partial(\Delta x, \Delta y, \Delta z)}{\partial\left(\Delta V_{x x}, \Delta V_{y y}, \Delta V_{h}\right)}\right|$ is the Jacobian of the transformation (4) and $\widetilde{\boldsymbol{A}}=\overline{\boldsymbol{A}}^{-1}$. As this transformation is linear, it can be written as follows:

$$
J=|\operatorname{det} \widetilde{\boldsymbol{A}}|=\frac{r^{17}}{108 x y z\left(r^{2}-5 z^{2}\right)},
$$

and the matrix $\bar{A}$ inversion gives us:

$$
\widetilde{\boldsymbol{A}}=\frac{r^{5}}{6}\left[\begin{array}{ccc}
-\frac{1}{x} \frac{3 r^{2}-5 y^{2}}{r^{2}-5 z^{2}} & \frac{1}{x} \frac{r^{2}-5 x^{2}}{r^{2}-5 z^{2}} & \frac{1}{x} \frac{r^{2}-5 x^{2}}{r^{2}-5 z^{2}} \\
\frac{1}{y} \frac{r^{2}-5 y^{2}}{r^{2}-5 z^{2}} & -\frac{1}{y} \frac{3 r^{2}-5 x^{2}}{r^{2}-5 z^{2}} & \frac{1}{y} \frac{r^{2}-5 y^{2}}{r^{2}-5 z^{2}} \\
0 & 0 & \frac{1}{z}
\end{array}\right] .
$$

The direct integration of Eq. (5) allows to join $\sigma_{x}, \sigma_{y}, \rho$ parameters with $\widetilde{A}_{i k}$ components:

$$
\begin{gathered}
\int P_{\Delta V h}\left(\Delta V_{x x}, \Delta V_{y y}, \Delta V_{h}\right) \mathrm{d} \Delta V_{h}=\frac{J}{2 \pi C_{0} \sigma_{s}^{2}} \exp \\
\times\left[-\frac{C_{1}^{2} \Delta V_{x x}^{2}+C_{2}^{2} \Delta V_{y y}^{2}-2 C_{12} \Delta V_{x x} \Delta V_{y y}}{2 C_{0}^{2} \sigma_{s}^{2}}\right],
\end{gathered}
$$

where

$$
\begin{aligned}
C_{0}^{2} & =\widetilde{A}_{13}^{2}+\widetilde{A}_{23}^{2}+\widetilde{A}_{33}^{2}, \\
C_{1}^{2} & =\widetilde{A}_{21}^{2} \widetilde{A}_{33}^{2}+\widetilde{A}_{11}^{2} \widetilde{A}_{33}^{2}+\widetilde{A}_{11}^{2} \widetilde{A}_{23}^{2}+\widetilde{A}_{13}^{2} \widetilde{A}_{21}^{2}-2 \widetilde{A}_{11} \widetilde{A}_{13} \widetilde{A}_{21} \widetilde{A}_{23}, \\
C_{2}^{2} & =\widetilde{A}_{22}^{2} \widetilde{A}_{33}^{2}+\widetilde{A}_{12}^{2} \widetilde{A}_{33}^{2}+\widetilde{A}_{12}^{2} \widetilde{A}_{23}^{2}+\widetilde{A}_{13}^{2} \widetilde{A}_{22}^{2}-2 \widetilde{A}_{12} \widetilde{A}_{13} \widetilde{A}_{22} \widetilde{A}_{23}, \\
C_{12} & =-\widetilde{A}_{11} \widetilde{A}_{12} \widetilde{A}_{23}^{2}-\widetilde{A}_{21} \widetilde{A}_{22} \widetilde{A}_{13}^{2}-\widetilde{A}_{11} \widetilde{A}_{12} \widetilde{A}_{33}^{2}-\widetilde{A}_{21} \widetilde{A}_{22} \widetilde{A}_{33}^{2} \\
& +\widetilde{A}_{11} \widetilde{A}_{13} \widetilde{A}_{22} \widetilde{A}_{23}+A_{12} \widetilde{A}_{13} \widetilde{A}_{21} \widetilde{A}_{23} .
\end{aligned}
$$


Comparing (7) with (3) results in:

$$
\begin{aligned}
\rho & =\frac{C_{12}}{C_{1} C_{2}}, \\
\sigma_{x} & =\frac{1}{\sqrt{1-\rho^{2}}} \frac{C_{0}}{C_{1}} \sigma_{s}, \\
\sigma_{y} & =\frac{1}{\sqrt{1-\rho^{2}}} \frac{C_{0}}{C_{2}} \sigma_{s} .
\end{aligned}
$$

Inserting components of matrix (6) to (8) and (9) yields:

$$
\begin{aligned}
\rho & =\frac{z^{4}-y^{2} z^{2}-x^{2} z^{2}-2 y^{4}+x^{2} y^{2}-2 x^{4}}{z^{4}+2 x^{2} z^{2}+4 y^{4}+x^{4}}, \\
\frac{\sigma_{x}^{2}}{\sigma_{s}^{2}} & =\frac{\left(x^{2}+y^{2}\right)\left(y^{2}+z^{2}\right)\left(z^{2}+x^{2}\right)-x^{2} y^{2} z^{2}}{z^{4}+2 x^{2} z^{2}+4 y^{4}+x^{4}}\left(1-\rho^{2}\right)^{-1}, \\
\sigma_{y} & =\sigma_{x} .
\end{aligned}
$$

The symbolic calculations was partially supported by the Maxima Algebra System.

It is clear that it will not always be possible to determine which specific charge has been shifted, but explicit expressions of distribution parameters (10) allow us to reduce the number of fitting parameters because $\sigma_{y}=\sigma_{x}=\sigma$. Thus for diagonal EFG tensor components, taking into account distribution (3), we can write:

$$
\begin{aligned}
P_{V}\left(V_{x x}, V_{y y}\right)= & \frac{1}{2 \pi \sigma^{2} \sqrt{1-\rho^{2}}} \exp \left\{-\frac{1}{2 \sigma^{2}\left(1-\rho^{2}\right)}\right. \\
& \left.\times\left[\left(V_{x x}-V_{x x}^{(0)}\right)^{2}+\left(V_{y y}-V_{y y}^{(0)}\right)^{2}-2 \rho\left(V_{x x}-V_{x x}^{(0)}\right)\left(V_{y y}-V_{y y}^{(0)}\right)\right]\right\},
\end{aligned}
$$

where $V_{x x}^{(0)}$ and $V_{y y}^{(0)}$ are the values of unperturbed EFG tensor components.

\section{Distribution $P\left(v_{z z}, \eta\right)$}

Let us notice that $V_{z z}$ and $\eta$ are parameters that completely define the occurrence of powder NMR signals. Particularly, after the diagonalization of the EFG tensor, $V_{z z}$ is not necessarily the "third" component, and according to the convention $\left|V_{z z}\right| \geqslant\left|V_{y y}\right| \geqslant\left|V_{x x}\right|$, EFG tensor requires re-arrangement. Thus, in that case $V_{z z}$ is not a component but positive parameter, while in the previous section $V_{x x}$ and $V_{y y}$ are true EFG tensor components. Dependencies between $V_{z z}, \eta$ and $V_{x x}, V_{y y}$ are shown in Fig. 2. 


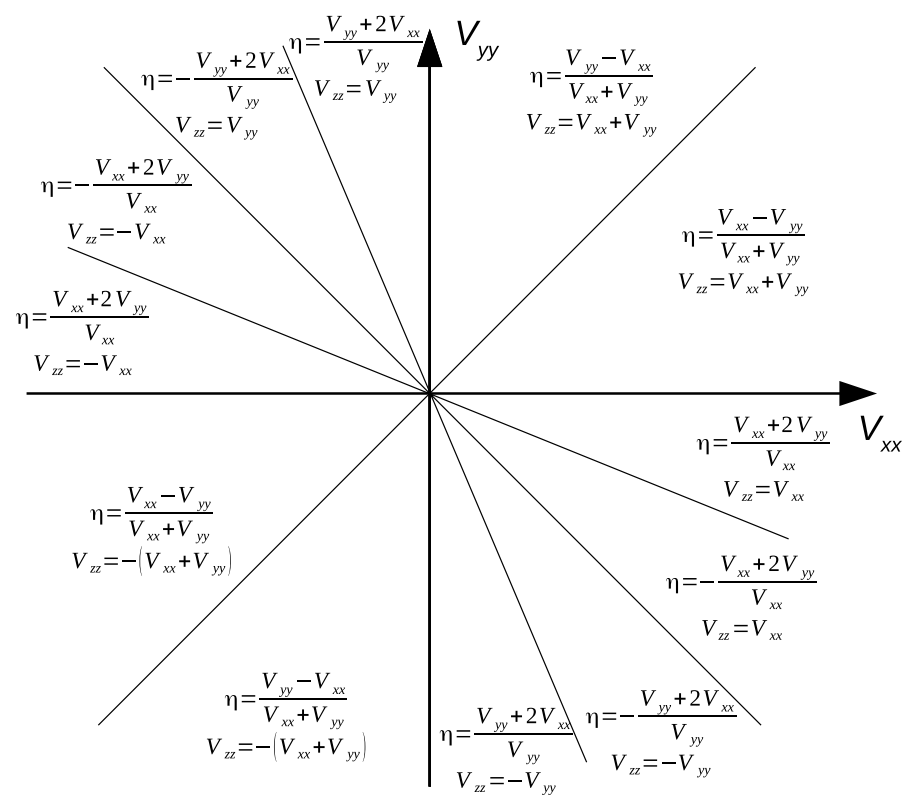

Fig. 2 Schematic representation of dependences between $V_{z z}, \eta$ and $V_{x x}, V_{y y}$

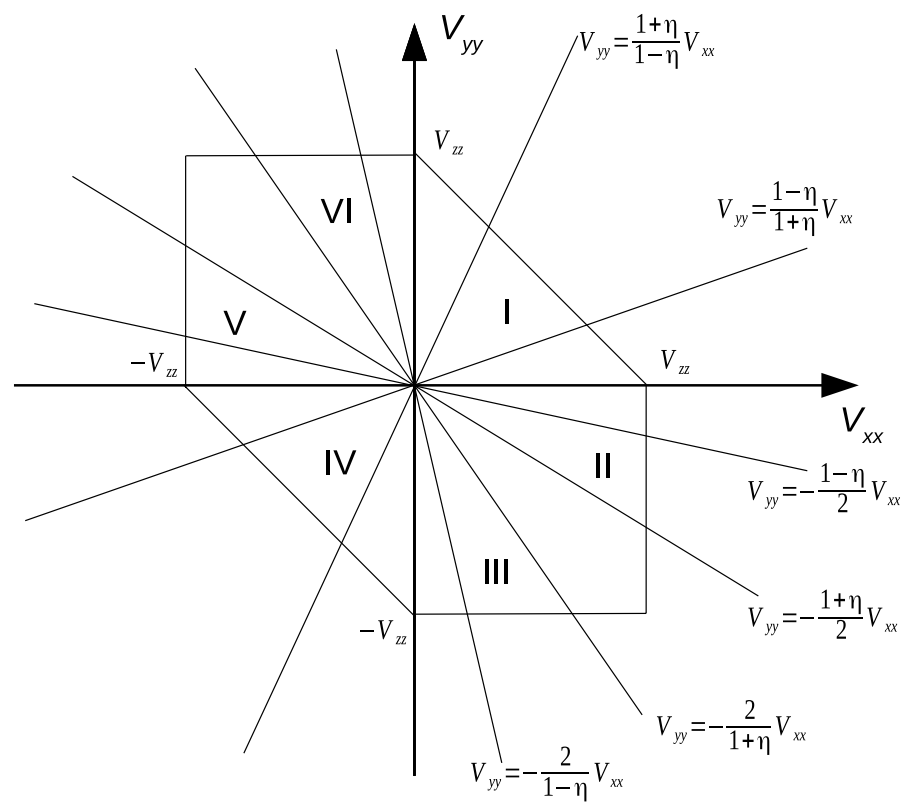

Fig. 3 Six integration areas corresponding to the condition $f\left(V_{x x}, V_{y y}\right) \leq\left(V_{z z}, \eta\right)$

The function $f: \mathbb{R}^{2} \rightarrow \mathbb{R}^{2}$ that defines transformation $\left(V_{z z}, \eta\right)=f\left(V_{x x}, V_{y y}\right)$ maps two to two variables. However, it is not possible to find a distribution $P\left(V_{z z}, \eta\right)$ in 
the same way as $P_{V}$ (through the Jacobian), because $f$ has no unambiguous inverse transformation. Therefore, it is necessary to use a general method of densities transformation [13]:

$$
P\left(V_{z z}, \eta\right)=\frac{\partial}{\partial V_{z z}} \frac{\partial}{\partial \eta} \int_{f\left(V_{x x}, V_{y y}\right) \leq\left(V_{z z}, \eta\right)} P_{V}\left(V_{x x}, V_{y y}\right) \mathrm{d} V_{x x} \mathrm{~d} V_{y y}
$$

The scheme of integration areas corresponding to condition $f\left(V_{x x}, V_{y y}\right) \leq\left(V_{z z}, \eta\right)$ is shown by Fig. 3.

Because direct integration of distribution $P_{V}\left(V_{x x}, V_{y y}\right)$ in the form given in (11) over all six areas is very complicated, let us consider some special cases.

\section{$5.1 v_{z z}^{(0)}=0$}

The case where both $V_{x x}^{(0)}=0$ and $V_{y y}^{(0)}=0$ corresponds to a regular structure in which there is no electric gradient in the quadrupolar nucleus. The asymmetry parameter is then undetermined. Because quadrupolar interactions are among the strongest in NMR, this situation is defect sensitive. Moreover, because there are no limitations to dispersion $\sigma$, the case $V_{z z}^{(0)}=0$ can be used with isotropic, amorphous solids.

The direct integration of (11) over all six areas gives:

$$
\begin{aligned}
& P\left(V_{z z}, \eta\right)=\frac{V_{z z}}{\pi \sigma^{2} \sqrt{1-\rho^{2}}} \\
& \times\left\{\exp \left[-\frac{V_{z z}^{2}}{4 \sigma^{2}} \frac{1}{1-\rho^{2}}\left(1+\eta^{2}-\left(1-\eta^{2}\right) \rho\right)\right]\right. \\
& +\exp \left[-\frac{V_{z z}^{2}}{2 \sigma^{2}} \frac{1}{1-\rho^{2}}\left(\left(\frac{1-\eta}{2}+\rho\right)^{2}+1-\rho^{2}\right)\right] \\
& \left.+\exp \left[-\frac{V_{z z}^{2}}{2 \sigma^{2}} \frac{1}{1-\rho^{2}}\left(\left(\frac{1+\eta}{2}+\rho\right)^{2}+1-\rho^{2}\right)\right]\right\} \text {. }
\end{aligned}
$$

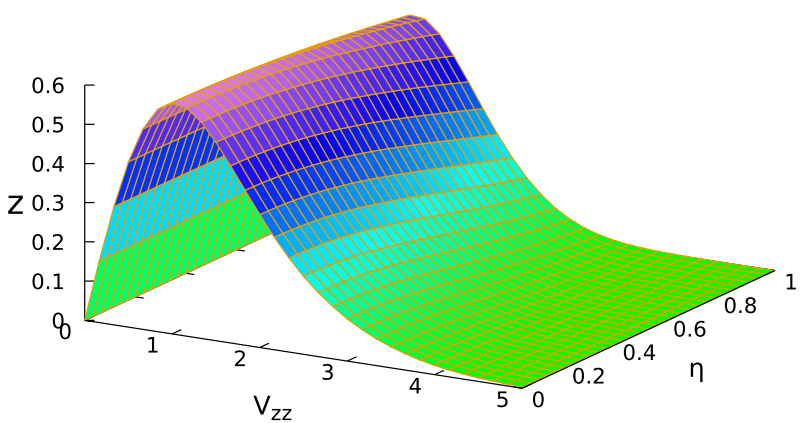

Fig. 4 Joint distribution $P\left(V_{z z}, \eta\right)$ for $V_{z z}^{(0)}=0, \rho=0$ and $\sigma=1$ 
The joint distribution (13) of $V_{z z}$ and $\eta$ in this case is presented in Fig. 4.

\section{$5.2 v_{z z}^{(0)} \gg 0$}

When $V_{z z}^{(0)} \gg 0$, then the density (11) can be integrated only over areas in which the distribution is substantially different from 0 . For $\eta$ significantly different from 1 , the integration can be done over area I only. Using transformation (12), the direct integration of (11) gives:

$$
\begin{aligned}
P\left(V_{z z}, \eta\right) \approx & \frac{V_{z z}}{4 \pi \sigma^{2} \sqrt{1-\rho^{2}}} \\
& \times\left\{\exp \left[-\frac{1}{4 \sigma^{2}}\left(\frac{\left(V_{z z} \eta-V_{z z}^{(0)} \eta^{(0)}\right)^{2}}{1-\rho}+\frac{\left(V_{z z}-V_{z z}^{(0)}\right)^{2}}{1+\rho}\right)\right]\right. \\
& \left.+\exp \left[-\frac{1}{4 \sigma^{2}}\left(\frac{\left(V_{z z} \eta+V_{z z}^{(0)} \eta^{(0)}\right)^{2}}{1-\rho}+\frac{\left(V_{z z}-V_{z z}^{(0)}\right)^{2}}{1+\rho}\right)\right]\right\} .
\end{aligned}
$$

When $\eta$ is close to 1 , an integration over area II should be added. In this case, the $P_{\text {add }}\left(V_{z z}, \eta\right)$ addition should be applied to the formula (14):

$$
\begin{aligned}
P_{a d d}\left(V_{z z}, \eta\right)= & \frac{V_{z z}}{4 \pi \sigma^{2} \sqrt{1-\rho^{2}}} \\
\times & \left\{\operatorname { e x p } \left[-\frac{1}{2 \sigma^{2}\left(1-\rho^{2}\right)}\left(\left(V_{z z}-V_{z z}^{(0)} \frac{1+\eta^{(0)}}{2}\right)^{2}\right.\right.\right. \\
& +\left(V_{z z} \frac{1-\eta}{2}-V_{z z}^{(0)} \frac{1-\eta^{(0)}}{2}\right)^{2} \\
- & \left.\left.2 \rho\left(V_{z z}-V_{z z}^{(0)} \frac{1+\eta^{(0)}}{2}\right)\left(V_{z z} \frac{1-\eta}{2}-V_{z z}^{(0)} \frac{1-\eta^{(0)}}{2}\right)\right)\right] \\
+ & \exp \left[-\frac{1}{2 \sigma^{2}\left(1-\rho^{2}\right)}\left(\left(V_{z z}-V_{z z}^{(0)} \frac{1+\eta^{(0)}}{2}\right)^{2}\right.\right. \\
& +\left(V_{z z} \frac{1+\eta}{2}-V_{z z}^{(0)} \frac{1-\eta^{(0)}}{2}\right)^{2} \\
- & \left.\left.\left.2 \rho\left(V_{z z}-V_{z z}^{(0)} \frac{1+\eta^{(0)}}{2}\right)\left(V_{z z} \frac{1+\eta}{2}-V_{z z}^{(0)} \frac{1-\eta^{(0)}}{2}\right)\right)\right]\right\} .
\end{aligned}
$$


Fig. 5 Joint distribution

$P\left(V_{z z}, \eta\right)$ for $V_{z z}^{(0)}=1, \rho=0$,

$\sigma=0.15$ and $\mathbf{a} \eta^{(0)}=0, \mathbf{b}$

$\eta^{(0)}=0.5, \mathbf{c} \eta^{(0)}=1$
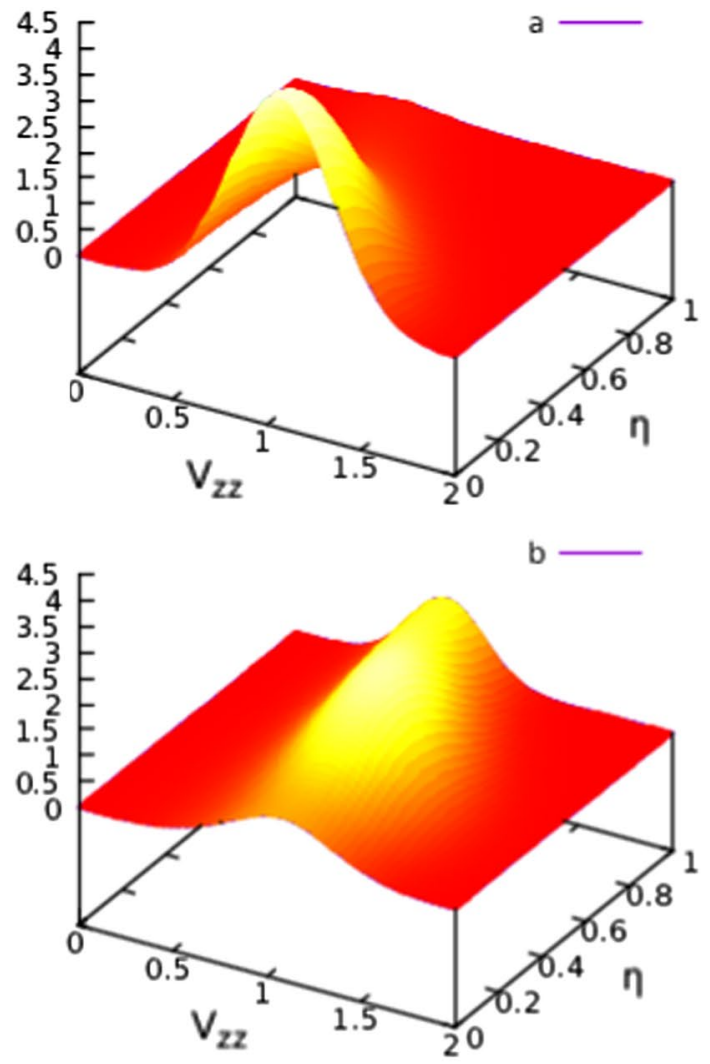

c $\longrightarrow$

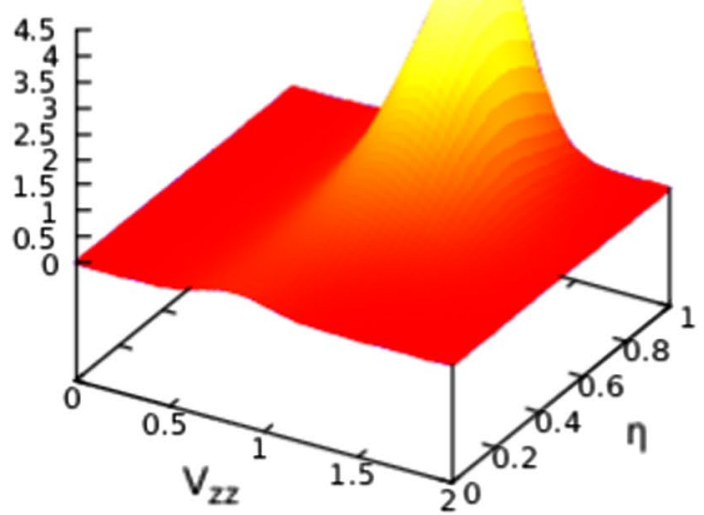

It should be noted that the formula (14) even with the above correction (15) is only approximate and it does not normalize to 1 . The sum of joint distributions (14) and (15) is shown in Fig. 5. 


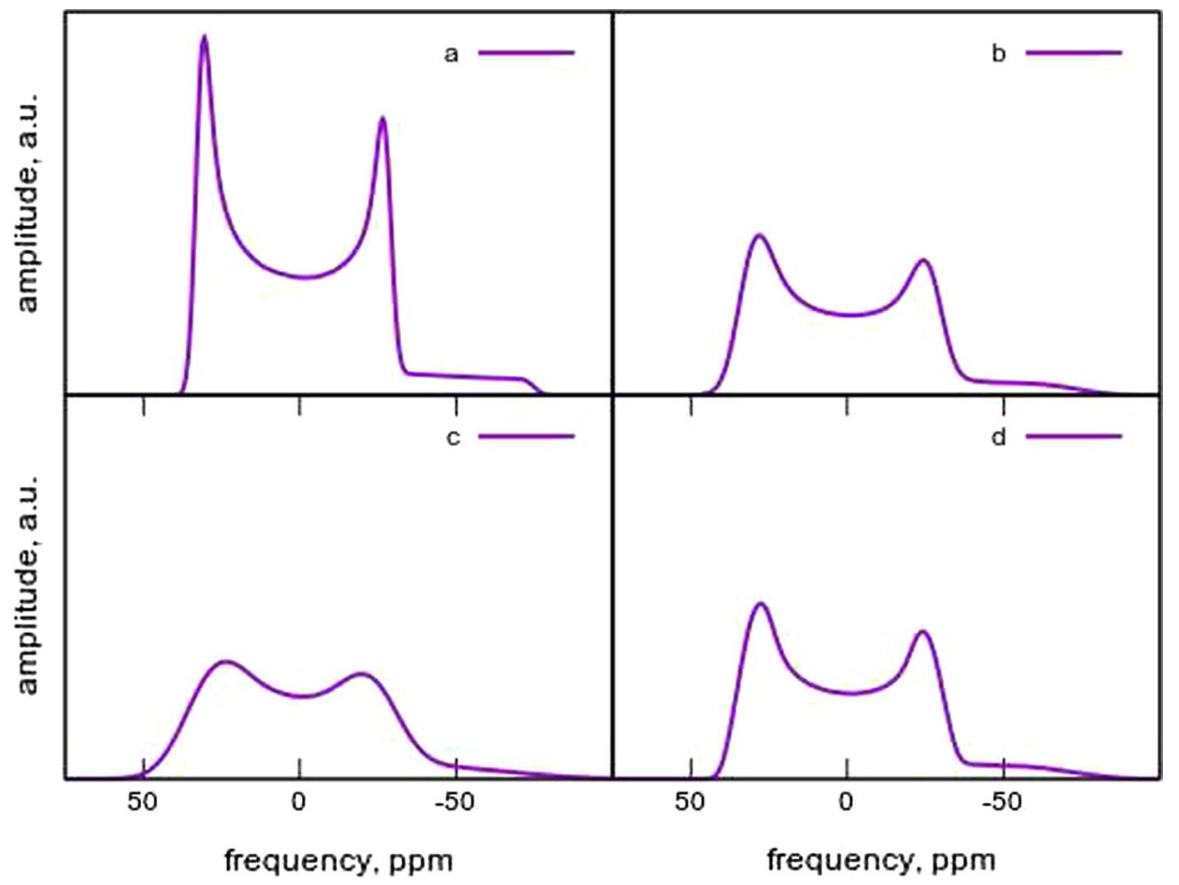

Fig. 6 Central transition MAS NMR spectra of quadrupolar nuclei for $\eta^{(0)}=0, V_{z z}^{(0)}=$ const and: a $\sigma=0, \rho=0, \mathbf{b} \sigma=0.08 V_{z z}, \rho=0, \mathbf{c} \sigma=0.16 V_{z z}, \rho=0, \mathbf{d} \sigma=0.08 V_{z z}, \rho=0,95$

\section{NMR Spectra}

The influence of dispersion of $V_{z z}$ and $\eta$ on NMR signals is shown by the example spectra in Fig. 6 for several dispersion parameters.

These spectra were obtained according to the theory presented in [1] for central transition (CT) NMR of quadrupolar nuclei in the magic angle spinning (MAS) regime by taking into account the dispersion (14) calculated in this work.

\section{Summary}

The joint dispersion of electric field gradient tensor components was made directly from the disturbance of the initial electric charge system surrounding the quadrupolar nucleus. In addition to that, the dependence between the structural and the obtained dispersion parameters was calculated, which resulted in the reduction of the number of dispersion parameters. The theory presented in this work can be useful, owing to the analytic form of the obtained distributions, for the modeling of NMR signals in disordered compounds with quadrupolar interactions where parameters are scattered around initial magnitudes. The influence 
of the obtained theory on NMR signals has been presented in this work with the example central transition MAS NMR spectra for several cases.

Open Access This article is distributed under the terms of the Creative Commons Attribution 4.0 International License (http://creativecommons.org/licenses/by/4.0/), which permits unrestricted use, distribution, and reproduction in any medium, provided you give appropriate credit to the original author(s) and the source, provide a link to the Creative Commons license, and indicate if changes were made.

\section{References}

1. D. Freude, in Encyclopaedia of Analytical Chemistry, ed. by R.A. Meyers (Wiley, Chichester, 2000), pp. 12188-12224

2. K. Seleznyova, N. Sergeev, M. Olszewski, P. Stępień, S. Yagupov, M. Strugatsky, J. Kliava, Solid State Nucl. Magn. Reson. 70, 38 (2015)

3. K. Seleznyova, N. Sergeev, M. Olszewski, P. Stępień, S. Yagupov, M. Strugatsky, J. Kliava, Solid State Nucl. Magn. Reson. 85-86, 12 (2017)

4. B.V. Padlyak, N.A. Sergeev, M. Olszewski, V.T. Adamiv, Ya.V. Burak, Phys. Chem. Glasses Eur. J. Glass Sci. Techn. B 55, 25 (2014)

5. A.M. Panich, A.I. Shames, N. Froumin, C.C. Tang, Y. Bando, Phys. Rev. B 72, 85307 (2005)

6. A.M. Panich, A.I. Shames, R. Rosentsveig, R. Tenne, J. Phys.: Condens. Matter 21, 395301 (2009)

7. G. Czjzek, J. Fink, F. Gotz, H. Schmidt, J.M.D. Coey, J.P. Rebouillat, A. Lienard, Phys. Rev. B: Condens. Matter 23, 2513 (1981)

8. G. Czjzek, Hyperfine Interact. 14, 189 (1983)

9. M. Maurer, Phys. Rev. B 34, 8996 (1986)

10. G. Le Caër, R.A. Brand, J. Phys.: Condens. Matter 10, 10715 (1998)

11. A. Abragam, The Principles of Nuclear Magnetism (Clarendon, Oxford, 1961)

12. W. Feller, An Introduction to Probability Theory and Its Applications (Wiley, New York, 1968)

13. L. Winterfeld, Transformation of Probability Densities. https://en.wikibooks.org. Accessed 4 July 2017 